\title{
Is there any different risk factor for clinical relevant pancreatic fistula according to the stump closure method following left-sided pancreatectomy?
}

\author{
Hyun Joo Yoo, Kwang Yeol Paik, and Ji Seon Oh
}

\author{
Department of Surgery, Yeouido St. Mary's Hospital, College of Medicine, The Catholic University of Korea, Seoul, Korea
}

\begin{abstract}
Backgrounds/Aims: Consistency on risk factors for postoperative pancreatic fistula (POPF) after left-sided pancreatectomy (LP) according to the stump closure methods has not been revealed. Appropriate surgical stump closure method after LP is still in debate. This study investigates risk factors for POPF according to the closure methods in LP. Methods: A total of 49 consecutive patients underwent LP with a stapler closure (ST) or hand-sewn closure (HS) between June 2001 and September 2016. The risk factors of pancreatic fistulas were investigated in 49 LPs according to stump closure methods, HS ( $n=19)$, and ST $(n=30)$. Results: There was no significant difference in the incidence of overall POPF (HS $42.1 \%$ vs. ST 50.0\%) and clinical relevant POPF (CR-POPF) (HS 5.3\% vs. ST 6.7\%) between two groups. In the ST group, the pancreas was significantly thick in patients with CR-POPF (27 mm vs. $17 \mathrm{~mm})$ and the tumor was also larger (58 mm vs. $27 \mathrm{~mm}$ ). In the HS group, the operation time was longer in CR-POPF group (515 min vs $292 \mathrm{~min})$. In univariate analysis, wider diameter of the pancreatic duct (27 mm vs $16 \mathrm{~mm}$ ) was associated with POPF in the HS group. There was no meaningful risk factor for POPF in the ST group. Conclusions: Incidence of overall POPF between the ST and HS group were clinically insignificant in this study. The thickness of the pancreas and the tumor diameter are factors significantly associated with CR-POPF in the ST group. Long operation time was the only factor associated with CR-POPF in the HS group. (Ann Hepatobiliary Pancreat Surg 2019;23:385-391)
\end{abstract}

Key Words: Risk; Pancreatic fistula; Pancreatectomy

\section{INTRODUCTION}

Pancreatic fistula after left-sided pancreatectomy (LP) is the surgeon's major concern as that in pancreatic fistula after pancreaticoduodenectomy. Although various techniques have been attempted to prevent the development of postoperative pancreatic fistula (POPF) after LP, no definite consensus has been established. ${ }^{1}$

Among various methods of stump closure after LP, stapler closure (ST) and hand-sewn (HS) are the most typical and popular. The stapler closure is the mainly selected method during laparoscopic left-sided pancreatectomy whereas the hand-sewn suture is the method commonly performed during laparotomy, regardless of reinforcement of the stump closure. The European multicenter trial (DISPACT) demonstrated that the incidence of POPF was comparable between stapler and hand-sewn closures. ${ }^{2}$

Including development of various closure methods, ongoing efforts for reducing pancreatic fistula have not been completed yet. In this context, identifying factors which affect the occurrence of the pancreatic fistula after LP is important. This research aimed to investigate the risk factor for POPF due to the closure methods during LP.

\section{MATERIALS AND METHODS}

49 patients underwent LP between 2001 and June 2016 were enrolled in the study. Surgery was performed by two surgeons (EK Kim, KY Paik) in a single institution.

Clinicopathological variables of 49 patients were collected retrospectively. Several variables related to intraoperative findings, including tumor size, pancreatic duct

Received: March 7, 2019; Revised: July 11, 2019; Accepted: July 25, 2019

Corresponding author: Kwang Yeol Paik

Department of Surgery, Yeouido St. Mary's Hospital, College of Medicine, The Catholic University of Korea, 10 63-ro, Yeongdeungpo-gu, Seoul 07345 , Korea

Tel: +82-2-3779-2232, Fax: +82-2-786-0802, E-mail: kpaik@outlook.com

Copyright (C) 2019 by The Korean Association of Hepato-Biliary-Pancreatic Surgery

This is an Open Access article distributed under the terms of the Creative Commons Attribution Non-Commercial License (http://creativecommons.org/ licenses/by-nc/4.0) which permits unrestricted non-commercial use, distribution, and reproduction in any medium, provided the original work is properly cited. Annals of Hepato-Biliary-Pancreatic Surgery • pISSN: 2508-5778 • elSSN: 2508-5859 
size, operation time, blood loss, resection of adjacent organs, the way of approach (laparoscopic vs. laparotomy), and pancreatic thickness at the stump were reviewed. At the same moment, variables focused on postoperative outcomes and POPF including pathologic diagnosis, serum and drain amylase were also evaluated.

All patients were preoperatively evaluated with contrast-enhanced abdominal computed tomography (CT) or abdominal magnetic resonance imaging (MRI). Somatostatin analog was routinely used until postoperative day 7 . The abdominal CT was performed 7 days after the operation to assess postoperative morbidity. The thickness of the pancreatic resection site was determined twice on the CT, pre- and postoperatively, to improve the degree of reliability.

For the ST method, the pancreas was transected using an Echelon 60 with gold cartridge (Johnson \& Johnson, Cincinnati, OH, USA) until 2012. Occasionally two cartridges were used for transection of the thick pancreas. After the year 2012, the Black type Endo GIA ${ }^{\text {TM }}$ Reloads with Tri-Staple ${ }^{\mathrm{TM}}$ (Covidien, North Haven, CT) was used for resection. Fibrin glue was routinely applied on the resection site. No additional reinforcement suture was done in laparoscopic cases.

The HS method mainly performed during laparotomy. For all of HS patients, the pancreas was transected carefully with a knife and following ligation of the main duct by using 3-0 PDS suture was performed. After transection, reinforcement of pancreas stump closure was performed via fish-mouth technique. In laparotomy, fibrin glue was also used after resection of the pancreas. Additonally, absorbable fibrin sealant patch (TachoSil; Nycomed Austria $\mathrm{GmbH}$, Linz, Austria) or absorbable polyglactin acid mesh sheet (Neoveil; Gunze Corp., Kyoto, Japan) were used in the past five years.

In cases of spleen-preserving distal pancreatectomy with excision of splenic vessels, short gastric arteries and veins were preserved, as reported by Warshaw. ${ }^{3}$ After separation, the distal pancreas was taken out through an extended incision of umbilical trocar site.

The amylase level of drain and serum were measured every day until the day of the drain removal. Since the year 2012, the level was only checked on the postoperative day 1,3,5 and 7 . The drain tube was removed when the amylase level of the drain reached to the normal range and if there was no evidence of POPF on the postoperative CT scan.

POPF was defined according to the International Study Group on Pancreatic Fistula Definition (ISGPF) as a drain output of on or after postoperative day 3 with an amylase value greater than 3 times the serum amylase. ${ }^{4}$ Three different grades of POPF (Grades A, B, C) were defined according to the patient's hospital course. All patients above Grade B were defined as clinical relevant POPF (CRPOPF).

Descriptive and comparative statistics were performed using SPSS 18 software. The value of continuous variables was reported as a median and interquartile range. Continuous variables were compared using the Student t-test or Mann-Whitney test, due to the appropriate type of distribution. Categorical variables were compared by using $\chi^{2}$ or the Fisher exact test depending on the number of observations. A $p$ value $\leq 0.05$ was considered signifi cant in all cases.

\section{RESULTS}

\section{Patients}

Table 1 shows the results of basic characteristics. The ST method was adopted for most of the laparoscopic LP patients $(n=30)$. The rest of 19 patients underwent open LP by using the HS method. The most common postoperative diagnosis was pancreatic ductal adenocarcinoma, present in $63 \%$ of the 49 patients, followed by cystic neoplasms $(15 \%)$ including intraductal papillary mucinous neoplasm; mucinous cystic neoplasm; serous cystadenoma; and solid pseudopapillary neoplasm and pancreatic neuroendocrine tumors $(12 \%)$.

Both groups had similar operation time and the HS group tended to show more bleeding. Conventional risk factors such as tumor size, pancreatic duct diameter, and thickness didn't show the difference. The ST method was more likely to be used in cases with a laparoscopic approach, a tumor located on the pancreas tail, spleen-preserving surgery, and pathologically benign findings.

\section{Overall POPF}

Table 2 describes the factors related to POPF and postoperative outcomes such as hospital stay. The overall fistula rate which now revised as the biochemical leak was 
Table 1. Clinicopathologic data of the cohort groups according to the closure method

\begin{tabular}{lcccc}
\hline & Overall (n=49) & ST (n=30) & HS (n=19) & $p$-value \\
\hline Age (year) & $57.2 \pm 16.2$ & $54.9 \pm 15.5$ & $60.7 \pm 17.1$ & 0.225 \\
Operation time (min) & $317.4 \pm 91.5$ & $324.1 \pm 98.1$ & $306.2 \pm 80.9$ & 0.542 \\
Expected blood loss (ml) & $948.4 \pm 750.2$ & $744.8 \pm 633.9$ & $1246.1 \pm 830.1$ & 0.062 \\
Tumor size (mm) & $30.9 \pm 20.2$ & $29.8 \pm 16.6$ & $33.5 \pm 27.3$ & 0.596 \\
P-duct size (mm) & $2.2 \pm 0.9$ & $2.2 \pm 1.0$ & $2.2 \pm 0.8$ & 0.957 \\
Pancreas thickness 1 (mm) & $13.2 \pm 5.6$ & $13.9 \pm 5.3$ & $11.6 \pm 6.2$ & 0.215 \\
Pancreas thickness 2 (mm) & $16.6 \pm 6.2$ & $17.8 \pm 6.4$ & $14.1 \pm 5.0$ & 0.075 \\
Case of pancreas tail lesion (\%) & $26(53.1 \%)$ & $22(73.3 \%)$ & $4(21.1 \%)$ & 0.001 \\
Laparoscopic approach (\%) & $25(51.0 \%)$ & $23(76.7 \%)$ & $2(10.5 \%)$ & 0.000 \\
Spleen-preserved (\%) & $21(42.9 \%)$ & $17(56.7 \%)$ & $4(21.1 \%)$ & 0.014 \\
Warshaw technique (\%) & $17(34.7 \%)$ & $14(46.7 \%)$ & $3(15.8 \%)$ & 0.026 \\
Pathologic benign lesion (\%) & $29(59.2 \%)$ & $21(70.0 \%)$ & $8(42.1 \%)$ & 0.051 \\
Combined resection (\%) & $5(10.2 \%)$ & $3(10.0 \%)$ & $2(10.5 \%)$ & 0.652 \\
Diameter of the main pancreatic duct $>3 \mathrm{~mm}(\%)$ & $10(20.4 \%)$ & $7(23.3 \%)$ & $3(15.8 \%)$ & 0.023 \\
\hline
\end{tabular}

Table 2. The factors related to POPF and postoperative outcomes according to the closure method

\begin{tabular}{lcccc}
\hline & Overall $(\mathrm{n}=49)$ & $\mathrm{ST}(\mathrm{n}=30)$ & HS $(\mathrm{n}=19)$ & $p$-value \\
\hline POPF (\%) & 46.9 & 50.0 & 42.1 & 0.669 \\
CR-POPF (\%) & 6.1 & 6.7 & 5.3 & 0.404 \\
JP amylase ${ }^{\text {POD1 }}(\mathrm{mg} / \mathrm{dl})$ & $6785.2 \pm 7992.5$ & $6696.3 \pm 6545.8$ & $6949.3 \pm 1045.7$ & 0.928 \\
Amylase $>5000^{\mathrm{POD} 1}(\%)$ & 34.7 & 40.0 & 26.3 & 0.528 \\
Hospital day & $10.6 \pm 3.9$ & $9.46 \pm 3.2$ & $12.7 \pm 4.3$ & 0.006 \\
\hline
\end{tabular}

$46.9 \%$ and clinical relevant fistula above grade B was $6.1 \%$. Both types of fistula did not show any significant differences between the ST and HS groups.

The level of drain amylase and the portion of patients who showed the level of amylase above 5000 on postoperative day 1 were also similar. The hospital stay of the ST group was significantly shorter than the HS group. Since the stapler was mostly used in laparoscopic cases, it might be the reason why hospital stay in HS group is longer than ST group.

Further subgroup analysis was performed for CR-POPF between two groups. In ST group, CR-POPF was associated with large sized tumor and the thick pancreas stump, which is measured at the postoperative abdominal CT.

Interestingly, CR-POPF did not occur in the laparoscopic group and patients who had benign pathology. In HS group, CR-POPF was associated with long operation time.

However, in multivariate analysis, we could not reveal any significant risk factor for clinical relevant fistula in both groups.

\section{Risk factors of POPF after left-sided pancreatectomy (ST)}

Univariate and multivariate analyses were used to reveal the risk factors for pancreatic fistulas after LP in the ST group. As shown in Table 3, the univariate analysis for risk factors was performed between the patients with CR-POPF ( $\mathrm{n}=2$ ) and without pancreatic fistulas or having grade A fistula $(n=28)$.

In the ST group, patients who had a large tumor and greater thickness measured on postoperative abdominal CT showed a statistically significant higher incidence of pancreatic fistula $(p<0.009$ and 0.024$)$. On the other hand, multivariate logistic regression analysis did not show significant differences for these two factors.

Combined resection of adjacent organ tends to have more cases of pancreatic fistula, even though it did not show statistical difference $(p=0.051)$. Pancreatic duct size, tumor location, and splenic preservation were not significant risk factors in both analyses. 
Table 3. Univariate analysis for the risk factors of CR-POPF in the ST group

\begin{tabular}{lccc}
\hline & CR-POPF (n=2) & Grade A/none (n=28) & $p$-value \\
\hline Age (year) & $65.0 \pm 25.5$ & $54.2 \pm 14.9$ & 0.349 \\
Operation time (min) & $240.0 \pm 0.0$ & $330.8 \pm 98.9$ & 0.214 \\
Expected blood loss (ml) & $1275.3 \pm 1732.1$ & $682.3 \pm 474.6$ & 0.221 \\
Tumor size (mm) & $58.5 \pm 12.2$ & $27.7 \pm 14.9$ & 0.009 \\
P-duct size (mm) & $3.4 \pm 1.7$ & $2.1 \pm 0.9$ & 0.083 \\
Pancreas thickness 1 (mm) & $18.0 \pm 2.8$ & $13.7 \pm 5.3$ & 0.261 \\
Pancreas thickness 2(mm) & $27.5 \pm 13.4$ & $17.1 \pm 5.4$ & 0.024 \\
Location, tail (\%) & 0.0 & 25.0 & 0.419 \\
Laparoscopy (\%) & 0.0 & 82.1 & 0.008 \\
Splenic preservation (\%) & 0.0 & 60.7 & 0.094 \\
Warshaw technique (\%) & 0.0 & 50.0 & 0.171 \\
Pathology benign (\%) & 0.0 & 25.0 & 0.025 \\
Combined resection (\%) & 50.0 & 7.1 & 0.051 \\
Diameter of the main pancreatic duct $>3 \mathrm{~mm} \mathrm{( \% )}$ & 50.0 & 25.0 & 0.643 \\
\hline
\end{tabular}

Table 4. Univariate analysis for the risk factors of CR-POPF in the HS group

\begin{tabular}{lccc}
\hline & CR-POPF (n=1) & Grade A/none (n=18) & $p$-value \\
\hline Age (year) & $82.0 \pm 0.0$ & $54.2 \pm 14.9$ & 0.349 \\
Op time (min) & $515.0 \pm 0.0$ & $330.8 \pm 98.9$ & 0.03 \\
EBL (ml) & $2500.0 \pm 0.0$ & $682.3 \pm 474.6$ & 0.119 \\
Tumor size (mm) & $2.5 \pm 0.0$ & $34.2 \pm 28.3$ & 0.762 \\
P-duct size (mm) & $3.2 \pm 0.0$ & $2.2 \pm 8.6$ & 0.268 \\
Pancreas thickness 1 (mm) & $5.0 \pm 0.0$ & $12.1 \pm 4.4$ & 0.284 \\
Pancreas thickness 2(mm) & $5.0 \pm 0.0$ & $14.8 \pm 4.4$ & 0.055 \\
Location_body (\%) & 10.0 & 44.4 & 0.279 \\
Laparoscopy (\%) & 0.0 & 11.1 & 0.725 \\
Splenic preservation (\%) & 0.0 & 22.2 & 0.458 \\
Pathology benign (\%) & 0.0 & 44.4 & 0.381 \\
Combined resection (\%) & 0.0 & 11.1 & 0.725 \\
Diameter of the main pancreatic duct $>3 \mathrm{~mm} \mathrm{( \% )}$ & 100.0 & 44.4 & 0.279 \\
\hline
\end{tabular}

\section{Risk factors of POPF after left-sided pancreatectomy (HS)}

As shown in Table 4, the univariate analysis for risk factors was performed between the patients with CR-POPF $(n=1)$ and without pancreatic fistulas or having grade A fistula $(n=18)$ in the HS group.

In the HS group, patients with a long operation time had a statistically significant high incidence of pancreatic fistulas $(p<0.03)$. Same as the ST group, multivariate logistic regression analysis did not show significant different for the operation time.

Interestingly in the HS group, the pancreas with little thickness which is measured on postoperative abdominal CT tends to have more cases of pancreatic fistula. Pancreatic duct size, tumor location, and splenic preservation were not significant risk factors in both analyses.

\section{DISCUSSION}

Perhaps the most interesting finding of our study was the higher rate of POPF occurrence after LP. As shown above, nearly half of patients experienced an increased level of drain amylase over postoperative day 3 . But we have to focus on only 4 patients $(6.1 \%)$ was required for re-intervention for internal and external drainage. None of them underwent morbidities such as intraabdominal bleeding or mortality.

The incidence of POPF after distal pancreatectomy (DP) has been reported to range from 18.6 to $64.9 \% .^{5-7}$ During postoperative day 30 , more than a third of patients 
have a pancreatic fistula after the procedure and a quarter of patients suffered from severe complications, according to the consensus classification of The International Study Group for Pancreatic Surgery (ISGPS). ${ }^{4}$

In the year 2017 new definition of the pancreatic fistula was introduced by ISGPS, ${ }^{8}$ and they only confined the fistula only as clinically relevant one such as 'Grade B and C'. The portion of $6.1 \%$ of CR-POPF in our study without mortality might be in the acceptable range.

In terms of the pancreatic head resection, the pancreas itself has been believed as a potentially important factor which can affect POPF occurrence. Besides, procedure-related factors such as the anastomotic methods did not arouse interest in its influence. Other procedure-related factors, such as management of pancreatic stump after DP was the major concern of surgeons in the matter of POPF. As the concept of minimally invasive surgery in pancreatectomy is in the limelight, stapler closure is the widely used standard technique for stump closure. ${ }^{9-12}$ However, appropriate surgical stump closure method after LP is still in debate.

There have been three meta-analyses of techniques for closure of the pancreatic remnant after DP and they did not show a statistically significant reduction in the incidence of pancreatic fistulas. ${ }^{13-15}$ DISPACT, A multicenter randomized controlled trial, published in 2014, has been evaluated the possibility of stapler closure to reduce POPF over hand-sewn closure after DP. The paper also concluded stapler closure did not reduce the incidence of POPF after DP compared to hand-sewn closure. ${ }^{2}$ As we can see, including DISPACT trial, most of current studies are focused on the association between POPF and stump closure methods.

As benign to borderline malignancies are mainly indicated for minimally invasive pancreatectomy including laparoscopy, we tried to analyze POPF risk factors separately due to the way of approach and stump closure methods.

In our study, the stapler closure method was mainly applied in laparoscopic surgery, whereas the hand-sewn method was common in open surgery such as radical antegrade modular pancreatectomy and tail resection (RAMPS). We expected there might be difference between the risk factors of POPF in subgroup analysis according to different stump closure method.
Our study proved the tumor size and the thickness of pancreas at the transection line are associated with CRPOPF patients in the ST group. In the HS group, long operation time was observed in CR-POPF patients. Moreover, in univariate analysis, larger diameter of main pancreatic duct was associated with POPF.

The well-known major risk for POPF following DP in the ST method is the thickness of pancreas. This reveals thick pancreas stump is vulnerable to POPF occurrence. ${ }^{16,17}$ Kawai et al. ${ }^{17}$ reported an increased thickness of the pancreas significantly affects the incidence of pancreatic fistulas after DP with stapler closure. They demonstrates 12-mm as a cutoff value for predicting POPF after DP whereas Okano et al. ${ }^{18}$ suggested $16-\mathrm{mm}$ as an appropriate cut-off value. Otherwise, Kim et al. ${ }^{16}$ identified $15 \mathrm{~mm}$ was division point between thin and thick pancreas. The main reason why the thickness is important is that thick pancreatic parenchyma easily torn with compression during stapler closure. ${ }^{17}$ Also leakage of pancreatic juice from the small duct might be another reason, which is so-called as 'tear' of the pancreas.

To improve the degree of reliability and reduce the measurement bias, we measured the thickness of pancreatic resection site twice, pre-and postoperatively. There was no significant difference between pre-and postoperative measured value.

It is well known that texture of the pancreas is one of major risk factor in POPF. Since the texture of pancreas generally not affected in the resection of the distal pancreas, it was not routinely monitored during current study. ${ }^{19-21}$ The texture of pancreas mostly related to the occurrence of POPF after pancreaticoduodenectomy.

On the literature, spleen-preservation is mentioned as another risk factor significantly associated with POPF after DP. ${ }^{22}$ It might occur because of the possibility of potential devascularization of the remnant pancreas in splenic preservation, which could cause failure in healing of the pancreatic stump. Ridolfini et al. ${ }^{23}$ showed patients who underwent concomitant splenectomy showed lower incidence of CR-POPF. In year 2008, Goh et al. ${ }^{24}$ announced similar results with analysis of 232 patients.

The mechanism of POPF formation in the hand-sewn group during DP was thought to be related to involvement of ischemic necrosis of the sutured surgical stump. CRPOPF also observed in the patients who had long oper- 
ation time in HS group, ${ }^{17}$ which was consistent with our study finding. The factors associated with increased complexity of surgery including increased operation time to be associated with increased non-PF related complications. ${ }^{24}$

Including non-PF related complications, five complications occurred and the overall complication rate was $10.2 \%$. Two patients experienced ileus (4\%), one patient suffered from postoperative pneumonia (2\%) and two cases of wound seroma (4\%) occurred. There was no mortality.

Despite of small number of patients in this study, it was obvious that risk factors for CR-POPF are different according to stump closure methods. Having in mind about these different risk factors, surgeons should consider about the way of method to reduce POPF during the operation. Because there is no clear guideline for choosing closure techniques due to the texture and thickness of the pancreas, further randomized clinical trials are required.

To sum up, although around the half of patients (46.9\%) experienced POFP after LP, incidence of overall POPF between different stump closure methods were clinically insignificant in this study. The thickness of the pancreas and the tumor diameter are factors significantly associated with the incidence of CR-POPF after LP in the ST group. On the other hand, the long operation time was the only factor associated with CR-POPF in the HS group.

\section{REFERENCES}

1. Miyasaka Y, Mori Y, Nakata K, Ohtsuka T, Nakamura M. Attempts to prevent postoperative pancreatic fistula after distal pancreatectomy. Surg Today 2017;47:416-424.

2. Diener MK, Seiler CM, Rossion I, Kleeff J, Glanemann M, Butturini G, et al. Efficacy of stapler versus hand-sewn closure after distal pancreatectomy (DISPACT): a randomised, controlled multicentre trial. Lancet 2011;377:1514-1522.

3. Warshaw AL. Distal pancreatectomy with preservation of the spleen. J Hepatobiliary Pancreat Sci 2010;17:808-812.

4. Bassi C, Dervenis C, Butturini G, Fingerhut A, Yeo C, Izbicki J, et al.; International Study Group on Pancreatic Fistula Definition. Postoperative pancreatic fistula: an international study group (ISGPF) definition. Surgery 2005;138:8-13.

5. Fox AM, Pitzul K, Bhojani F, Kaplan M, Moulton CA, Wei AC, et al. Comparison of outcomes and costs between laparoscopic distal pancreatectomy and open resection at a single center. Surg Endosc 2012;26:1220-1230.

6. Nakamura M, Wakabayashi G, Miyasaka Y, Tanaka M, Morikawa T, Unno M, et al.; Study Group of JHBPS, JSEPS. Multicenter comparative study of laparoscopic and open distal pancreatectomy using propensity score-matching. J Hepatobiliary Pancreat Sci 2015;22:731-736.

7. Ricci C, Casadei R, Buscemi S, Taffurelli G, D'Ambra M, Pacilio CA, et al. Laparoscopic distal pancreatectomy: what fac- tors are related to the learning curve? Surg Today 2015;45:50-56. 8. Bassi C, Marchegiani G, Dervenis C, Sarr M, Abu Hilal M, Adham M, et al.; International Study Group on Pancreatic Surgery (ISGPS). The 2016 update of the International Study Group (ISGPS) definition and grading of postoperative pancreatic fistula: 11 years after. Surgery 2017;161:584-591.

9. Kajiyama Y, Tsurumaru M, Udagawa H, Tsutsumi K, Kinoshita Y, Akiyama H. Quick and simple distal pancreatectomy using the GIA stapler: report of 35 cases. Br J Surg 1996;83:1711.

10. Okano K, Kakinoki K, Yachida S, Izuishi K, Wakabayashi H, Suzuki Y. A simple and safe pancreas transection using a stapling device for a distal pancreatectomy. J Hepatobiliary Pancreat Surg 2008;15:353-358.

11. Misawa T, Shiba H, Usuba T, Nojiri T, Uwagawa T, Ishida $Y$, et al. Safe and quick distal pancreatectomy using a staggered six-row stapler. American J Surg 2008;195:115-118.

12. Ferrone CR, Warshaw AL, Rattner DW, Berger D, Zheng $\mathrm{H}$, Rawal B, et al. Pancreatic fistula rates after 462 distal pancreatectomies: staplers do not decrease fistula rates. J Gastrointest Surg 2008;12:1691-1697; discussion 1697-1698.

13. Knaebel HP, Diener MK, Wente MN, Büchler MW, Seiler CM. Systematic review and meta-analysis of technique for closure of the pancreatic remnant after distal pancreatectomy. Br J Surg 2005;92:539-546.

14. Zhou W, Lv R, Wang X, Mou Y, Cai X, Herr I. Stapler vs suture closure of pancreatic remnant after distal pancreatectomy: a meta-analysis. American J Surg 2010;200:529-536.

15. Probst P, Hüttner FJ, Klaiber U, Knebel P, Ulrich A, Büchler MW, et al. Stapler versus scalpel resection followed by hand-sewn closure of the pancreatic remnant for distal pancreatectomy. Cochrane Database Syst Rev 2015;(11):CD008688.

16. Kim H, Jang JY, Son D, Lee S, Han Y, Shin YC, et al. Optimal stapler cartridge selection according to the thickness of the pancreas in distal pancreatectomy. Medicine (Baltimore) 2016;95:e4441.

17. Kawai M, Tani M, Okada K, Hirono S, Miyazawa M, Shimizu A, et al. Stump closure of a thick pancreas using stapler closure increases pancreatic fistula after distal pancreatectomy. American J Surg 2013;206:352-359.

18. Okano K, Oshima M, Kakinoki K, Yamamoto N, Akamoto S, Yachida S, et al. Pancreatic thickness as a predictive factor for postoperative pancreatic fistula after distal pancreatectomy using an endopath stapler. Surg Today 2013;43:141-147.

19. Pannegeon V, Pessaux P, Sauvanet A, Vullierme MP, Kianmanesh R, Belghiti J. Pancreatic fistula after distal pancreatectomy: predictive risk factors and value of conservative treatment. Arch Surg 2006;141:1071-1076; discussion 1076.

20. Yoshioka R, Saiura A, Koga R, Seki M, Kishi Y, Morimura R, et al. Risk factors for clinical pancreatic fistula after distal pancreatectomy: analysis of consecutive 100 patients. World J Surg 2010;34:121-125.

21. Sugimoto M, Gotohda N, Kato Y, Takahashi S, Kinoshita T, Shibasaki $\mathrm{H}$, et al. Risk factor analysis and prevention of postoperative pancreatic fistula after distal pancreatectomy with stapler use. J Hepatobiliary Pancreat Sci 2013;20:538-544.

22. Montorsi M, Zerbi A, Bassi C, Capussotti L, Coppola R, Sacchi M; Italian Tachosil Study Group. Efficacy of an absorbable fibrin sealant patch (TachoSil) after distal pancreatectomy: a multicenter, randomized, controlled trial. Ann Surg 2012;256:853-859; discussion 859-860.

23. Ridolfini MP, Alfieri S, Gourgiotis S, Di Miceli D, Rotondi F, Quero G, et al. Risk factors associated with pancreatic fistula after distal pancreatectomy, which technique of pancreatic stump closure is more beneficial? World J Gastroenterol 2007;13:50965100 . 
24. Goh BK, Tan YM, Chung YF, Cheow PC, Ong HS, Chan WH, et al. Critical appraisal of 232 consecutive distal pancreatectomies with emphasis on risk factors, outcome, and management of the postoperative pancreatic fistula: a 21-year experience at a single institution. Arch Surg 2008;143:956-965. 